\title{
Discovery of a potent angiotensin converting enzyme 2 inhibitor from Chinese Medicinal and Edible Plant via Docking-based Virtual Screening
}

Cheng-Ting Zi ( $\nabla$ zichengting@126.com )

Yunnan Agricultural University

Ning Zhang

Yunnan Agricultural University

Liu Yang

Chinese Academy of Sciences

Li-Xia Wang

Yunnan Agricultural University

Yi-Long Wu

Yunnan Agricultural University

Yin-Shan Su

Yunnan Agricultural University

Xuan-Jun Wang

Yunnan Agricultural University

\section{Research Article}

Keywords: COVID-19, angiotensin converting enzyme 2 (ACE2), drug discovery,virtual screening, molecular docking

Posted Date: June 4th, 2020

DOI: https://doi.org/10.21203/rs.3.rs-32515/v1

License: (c) (1) This work is licensed under a Creative Commons Attribution 4.0 International License. Read Full License 


\section{Abstract}

COVID-19 has outbreaked in Wuhan city, Hubei province of China since December $30^{\text {th }} 2019$, and spread nationwide and widely spilled over to other countries around the world that has been declared a public health emergency. However, there is no specific drug for the treatment of the disease. Therefore, identifying effective antiviral drugs to combat the disease is urgently needed. Angiotensin converting enzyme 2 (ACE2) has become the promising target to discovery new antiviral drugs to treat COVID-19, we have attempted to discover novel ACE2 inhibitors through ligand-based virtual screening. Finally, eight compounds were selected and tested ACE2 kinase inhibitory assay using fluorescence assays method. The results showed that four compounds (monoammonium glycyrrhizinate, glycyrrhizic acid methyl ester, ginsenoside Rg6 and ginsenoside F1) from 101 kinds of Chinese medicinal and edible plants which could inhibit ACE2 activity in vitro. Further efforts on chemical modification of these lead compounds are undergoing can lead to discover better agents against COVID-19.

\section{Introduction}

The 2019 novel coronavirus (2019-nCoV), SARS-CoV-2, was firstly reported on December $30^{\text {th }}$ in Wuhan city, Hubei province of China [1]. The disease was recently named as COVID-19 by the World Health Organization (WHO). COVID-19 has been spread nationwide and widely spilled over to other countries around the world has posed a serious global public health emergency [2]. According to WHO on March $15^{\text {th }} 2020$, there has been over outbreak of COVID-19 in 152 counties, leading to 173344 confirm cases and including 7019 deaths. However, there is no specific drug against COVID-19. Therefore, developing effective antiviral agents to combat the disease is urgently needed.

Up to date, researchers reported that the Cryo-EM structure of COVID-19 and the crystal structure of COVID-19 spike receptor $(S)$ binds angiotensin converting enzyme 2 (ACE2) complex [2-5]. Similar with syndrome-related coronavirus (SAR-CoV), $S$ protein of COVID-19 contains the receptor binding domain (RBD) binds to the ACE2 (Figure 1A-1B) [6]. The inhibitor of ACE2 is likely to change the conformation of ACE2 and block the interaction between $S$ protein and ACE2 receptor. Thus, ACE2 has become the promising target to discovery new antiviral drugs to treat COVID-19, and some inhibitors targeting ACE2, such as MLN-4760, DX600, 4S-16659 and NAAE [7-10]. However, due to its undesirable side effects, such as renal failure, persistent cough and angioedema, attempts to use the synthetic ACE2 inhibitors in clinic have been mostly unsuccessful. Therefore, more efforts are made for safe and specific drugs development.

Natural products from plant have served as important sources of lead compounds for developing drugs. Notably, many studies found that Traditional Chinese Medicine (TCM) extract effectively inhibited the activity of ACE $[11,12]$. Moreover, medicinal and edible plants are safe and effective play an important role in supporting healthcare both in China and around the world. In this study, we used docking 
screening to identify more natural ACE2 inhibitors from 101 kinds plants that are used in China medicine and as food supplements, therefore providing a potential prevention/treatment agents against COVID-19.

\section{Results And Discussion}

\subsection{Virtual Screening}

Computational approach comprising virtual screening, molecular docking and molecular dynamics (MD) simulation is a widely used method for the exploration of novel inhibitors against a target protein [13, 14], and cross docking is an approach to find the best structures for protein have been used in many studies [15-17]. Thus, the best crystal structure was selected for further study. In this paper, the crystal structures of ACE2 were obtained from RCSB Protein Data Bank (http://www.rcsb.org/pdb/home/home.do) and reported in the literatures $[2,4,18]$. DiscoveryStudio 4.0 software was used to the preparation of ligand and receptor. A comparison of the structures of ACE2 (2019-nCoV-RBD-ACE2) and 1R4L indicated that ACE2 (2019-nCoV-RBD-ACE2) [2] was most similarity with 1R4L (see Supplementary data Figure S1). The active site was defined by ligand of ACE2 complex (1R4L). Ligand (MLN-4760) was docked in the active site of ACE2 (2019-nCoV-RBD-ACE2) and ACE2 (1R4L) (Figure 1C). Best docking pose was output on the basis of glide score (best docking energy), root-mean-square deviation (RMSD) and docking binding affinity $\left(K_{i}\right)$ were calculated between the bioactive and docking conformations (Table 1). As shown in Table 1, RMSD values were less $0.3 \mathrm{~nm}$ and $K_{i}$ values were same order of magnitude as reported biological activity. It was suggested that ACE2 (2019-nCoV-RBD-ACE2) was the most suitable one.

Virtual screening represents a fast approach to identify novel hit structures and has been widely employed in modern drug discovery campaign. In our efforts to identify novel ACE2 inhibitors, we virtually screened (see Supplementary data Table S1.) 565 compounds from 101 kinds of Chinese medicinal and edible plants utilizing AutoDock Vina (v.1.0.2.) [19]. By applying a docking score cutoff of $\geq 9.0 \mathrm{kcal} / \mathrm{mol}$, we identified 33 compounds ( 5\%) with the maximum scores (Table 2). These compounds were used for further assessing the physiochemical.

\subsection{Physiochemical Properties}

To further screen anti-2019-nCoV agents from 33 compounds ( 5\%), the physiochemical properties of the identified compounds were calculated by MarvinSketch version 5.3.8 [20], including molecular weight (MW), molecular surface area (MSA), polar surface area (PSA), relative polar surface area (\%PSA), calculated partition coefficient (ClogP), calculated distribution coefficient at pH 7.4 (ClogD7.4), hydrogen bond donor (HD), hydrogen bond acceptor (HA), and rotatable bond (RB) (Table 3). For compounds (ZN00013, ZN00061, ZN00070, ZN00325, ZN00339, ZN00378, ZN00441 and ZN00451) had MW ranges between $426-821 \mathrm{~g} / \mathrm{mol}$, PSA were with the $9.23-256.0 \AA^{2}$ range, $\operatorname{ClogP}<5, C \log D_{7.4}<5$, with 1-7 HD and $\mathrm{RB} \approx 10$. Thus, 8 identified compounds matched Lipinski's rule [21, 22], which were selected for activity test. 
To assess the inhibitory potency of the identified compounds, eight compounds were subjected to in vitro ACE2 kinase inhibitory assay using Fluorescence assay method (see Supplementary data Supporting Text) $[7,23]$. The inhibition rate of those compounds was presented in Table 2. Interestingly, ZN00061 (monoammonium glycyrrhizinate), ZN00070 (glycyrrhizic acid methyl ester), ZN00441 (ginsenoside Rg6) and ZN00451 (ginsenoside F1) shown the most potent activity against ACE2 kinase among eight compounds. Ginsenoside Rg6 (inhibition rate: $81.62 \%$ ) and ginsenoside F1 (inhibition rate: $60.70 \%$ ) were isolated from ginseng $[24,25]$. Ginseng is one of the most commonly used traditional medicines in China, korea, Japan, and other Asian countries, shows various biological effects including anticancer, antioxidative, antiaging, neurovascular modulatory, antiviral, and other activities [26-29]. Ginsenoside Rg6 and ginsenoside F1 show antitumor, antioxidant and anti-inflammatory effects in vitro [30-33]. Previous study has reported that ginsenoside Rb1 exerted inhibitory activity against ACE [34]. This study could provide evidence for the biological function of gnsenosides at the molecular level. Monoammonium glycyrrhizinate (inhibition rate: $51.68 \%$ ) and glycyrrhizic acid methyl ester (inhibition rate: $50.40 \%$ ) were isolated from Glycyrrhiza uralensis Fisch (Glycyrrhiza uralensis F.) [35, 36]. Glycyrrhiza uralensis F., an ancient herbal medicine in traditional Chinese medicine, has been used for thousands of years. It has several important pharmacological activities, including anti-oxidant, anti-cancer, anti-inflammatory, antiulcer, anti-viral, and anti-HIV [37-39]. Monoammonium glycyrrhizinate and glycyrrhizic acid methyl ester have not been reported in the treatment of ACE2 related diseases. However, the results showed that two compounds had a certain inhibitory effect on ACE2 in vitro and could be used as a lead compounds for further study.

\subsection{Molecular Docking}

To further investigate the potential binding between ACE2 and the compounds, the molecular docking was performed. The complex structures for monoammonium glycyrrhizinate, glycyrrhizic acid methyl ester, ginsenoside Rg6 and ginsenoside F1 were presented in Figure 2. The key residues were labeled and the important molecular interaction including the hydrogen bonds (black dotted lines), hydrophobic interactions (red dotted lines), and salt bridges (yellow dotted lines) were summarized in Table 4. As shown in Figure 2A, it was observed that the complex was stabilized by three hydrogen bonds (with ASN63 and TYR-510), two hydrophobic interactions (with TYR-50 and PHE-504) and three salt bridges (with ASN-273 and HIS-345). The docking binding energy $(\Delta G)$ of the interaction and the corresponding docking binding affinity $\left(K_{\mathrm{d}}\right)$ were estimated to be $-1.63 \mathrm{kcal} / \mathrm{mol}$ and $6.23 \times 10^{2} \mathrm{M}^{-1}$, respectively. We found that glycyrrhizic acid methyl ester binds at the site of ACE2 by forming five hydrogen bonds (with SER-124, TYR-199, TRP-203, ASP-509 and TYR-510), two hydrophobic interactions with THR-125 and ASP-509, and two salt bridges with HIS-345 and LYS-187 (Figure 2B). The $\Delta G$ of stabilization was found to be $-1.16 \mathrm{kcal} / \mathrm{mol}, K_{\mathrm{d}}$ of $0.141 \mathrm{M}^{-1}$. Figure $2 \mathrm{C}$ showed that ginsenoside Rb1 was comfortably fitted at the active site cavity of ACE2. It interacted with SER-124, TYR-199, TRP-203, ASN-508, ASP-509 and TYR510 through hydrogen bonds, while TYR-202, ASN-508 and TYR-510 were involved in hydrophobic interactions. The complex was stabilized by a $\Delta G$ of $-4.72 \mathrm{kcal} / \mathrm{mol}$, which corresponded to a $K_{\mathrm{d}}$ of $3.45 \times$ $10^{4} \mathrm{M}^{-1}$. Ginsenoside F1 complex was stabilized by three hydrogen bonds with ASN51, and seven 
hydrophobic interactions with TYR-127, VAL-343, HIS-345 and PHE-504. The $\Delta G$ and $K_{\mathrm{d}}$ of ginsenoside F1 towards ACE2 were estimated to be $-1.84 \mathrm{kcal} / \mathrm{mol}$ and $4.49 \times 10^{2} \mathrm{M}^{-1}$, respectively. Based on the data, the presence of more hydrogen bonds in ginsenoside Rb1 seems the key factor for their high activity.

\section{Experimental}

\subsection{Materials}

Monoammonium glycyrrhizinate, glycyrrhizic acid methyl ester, ginsenoside Rg6 and ginsenoside F1 were purchased from Chengdu Proifa Technology Development Co., Ltd (Chengdu, China). Mca-R-P-PG-FS-A-F-K (Dnp)-OH was obtained from R\&D Systems (Minneapolis, MN). ACE2 protein was purchased from Sigma (St Louis, MO).

\subsection{Virtual Screening}

The docking screenings were conducted by using AutoDock Vina v.1.0.2. The docking parameters for AutoDock Vina were kept at their default values. The grid box was $40 \AA$ by $40 \AA$ by $40 \AA$, encompassing the catalytic pocket. The binding modes were clustered through the root mean square deviation (RMSD) among the Cartesian coordinates of the ligand atoms.

\subsection{Molecular Modeling}

DiscoveryStudio 4.0 software was used to the preparation of ligand and receptor. Autodock Tools v1.56 was used for grid and docking according to the literature [40]. Docking parameters were set as the defaults values, except "The Number of GA Runs" was set to 50 and maximum number of evals (medium) was set to 5,000,000 on AutoGrid v4.2.6 and AutoDock v4.2.6. Docking conformations were classified into different clusters by binding energy, and the cluster with the lowest binding energy was selected. In the selected cluster, conformations with the lowest binding energy and RMSD $(<2.0 \AA)$ were finally chosen to analyze the receptor-ligand interaction. Eetoposide was chosen as control ligand.

\subsection{Calculated Molecular Descriptors}

All compounds of were built and energy minimized by the Tripos force field with $0.05 \mathrm{kcal} /(\mathrm{mol} \AA)$. The Gasteiger-Huchel method was used to calculate charges. Energy minimization was performed by the Powell method with 2000 iterations. The calculated partition coefficient (ClogP) and polar surface area (PSA) were obtained from MarvinSketch version 5.3.8. (www.chemaxon.org) [41].

\subsection{In vitro ACE2 inhibitory activity assay}

In brief, ACE2 acitivity assay was performed using $10 \mu \mathrm{M}$ fluorogenic peptide substrate Mca-R-P-PG-F-SA-F-K (Dnp)-OH in balck 384-well opaque plates with $0.1 \%$ DMSO solution, $1 \mu \mathrm{M}$ captopril and $100 \mu \mathrm{M}$ test compounds. $0.2 \mathrm{ng} / \mu \mathrm{l}$ recombinant human ACE2 protein was added to the reaction mixtures. The fluorescence value was measured every other 2 minutes. The enzymatic activity was recorded on a 
SpectraMAX Gemini fluorescence spectrophotometer at an excitation wavelength of $328 \mathrm{~nm}$ and an emission wavelength of $392 \mathrm{~nm}$. Fluorescence was monitored at $36 \mathrm{~s}$ intervals for $15 \mathrm{~min}[7,17]$. The percentage of inhibition was calculated as follows:

$$
\text { Inhitition rate (\%) }=\left(1-\frac{S_{\mathrm{com}}-S_{\mathrm{con}}}{S_{\mathrm{DMsO}}-S_{\mathrm{con}}} \times 100 \text { where } S_{\text {com }}, S_{\text {con }}\right. \text { and }
$$
$S_{D M S O}$ were the slope value of the compound group, control group and DMSO group. The data represented the mean of three assay results.

\section{Conclusion}

In conclusion, we have discovered four compounds (monoammonium glycyrrhizinate, glycyrrhizic acid methyl ester, ginsenoside Rg6 and ginsenoside F1) from 101 kinds of Chinese medicinal and edible plants which could inhibit ACE2 activity in vitro. These compounds had strong potential to be further developed as novel ACE2 inhibitors. Further efforts on chemical modification of these lead compounds are undergoing can lead to discover better agents against COVID-19. CTMs are playing an important role in the treatment of COVID-19, Chinese medicinal and edible plants are usually more safe compared with CTMs, we will focus more on the validation of the identified compounds in cell lines and animals.

\section{Declarations}

\section{Acknowledgment}

This work was financial supported by the National Nature Science Foundation of China (Grant numbers 31960075, 21602196 and 31760226); the Yunnan Provincial Science and Technology Department (Grant numbers 2017ZF003, 2017FD084, and 2017FG001-046); Yunnan provincial key programs of Yunnan Eco-friendly Food International Cooperation Research Center project under grant (Grant number 2019ZG00909).

\section{Conflict of interest}

The authors declared no conflicts of interest.

\section{Open Access}

This article is distributed under the terms of the Creative Commons Attribution 4.0 International License (http://creativecommons.org/licenses/by/4.0/), which permits unrestricted use, distribution, and reproduction in any medium, provided you give appropriate credit to the original author(s) and the source, provide a link to the Creative Commons license, and indicate if changes were made.

\section{References}

1. CDC. 2019 Novel Coronavirus (2019-nCoV), Wuhan, China. (2020) 
2. J. Lan, J.W. Ge, J.F. Yu, S.S. Shan, H. Zhou, S.L. Fan, Q. Zhang, X.L. Shi, Q.S. Wang, L.Q. Zhang, X.Q. Wang, bioRxiv (2020)

3. D. Wrapp, N. Wang, K.S. Corbett, J.A. Goldsmith, C.L. Hsieh, O. Abiona, B.S. Graham, J.S. McLellan, Science 367, 1260-1263 (2020)

4. National Pathogen Resource Collection Center (NO. NMDCS0000001) (http://nmdc.cn/\#/resource/detail?no=NMDCS0000001)

5. R.H. Yan, Y.Y. Zhang, Y.N. Li, Y.Y. Guo, Q. Zhou, Science 367, 1444-1448 (2020)

6. F. Li, W. Li, M. Farzan, S.C. Harrison, Science 309, 1864-1868 (2005)

7. L.L. Huang, D.J. Sexton, K. Skogerson, M. Devlin, R. Smith, I. Sanyal, T. Parry, R. Kent, J. Enright, Q.L. Wu, G. Conley, D. DeOliveira, L. Morganelli, M. Ducar, C.R. Wescott, R.C. Ladner, J. Biol. Chem. 278, 15532-15540 (2003)

8. N.A. Dales, A.E. Gould, J.A. Brown, E. Calderwood, B. Guan, C.A. Minor, J.M. Gavin, P. Hales, V.K. Kaushik, M. Stewart, P.J. Tummino, C.S. Vickers, T.D. Ocain, M.A. Patane, J. Am. Chem. Soc. 124, 11852-11853 (2002)

9. M. Rella, C.A. Rushworth, J.L. Guy, A.J. Turner, T. Langer, R.M. Jackson, J. Chem. Inf. Model. 46, 708716 (2006)

10. M.J. Huentelman, J. Zubcevic, J.A. Hernández Prada, X.D. Xiao, D.S. Dimitrov, M.K. Raizada, D.A. Ostrov, Hypertension 44, 903-906 (2004)

11. J.M. Barbosa-Filho, V.K.M. Martins, L.A. Rabelo, M.D. Moura, M.S. Silva, E.V.L. Cunha, M.F.V. Souza, R.N.A. Almeida, I.A. Medeiros, Rev. Bras Farmacognosia. 16, 421-446 (2006)

12. X.D. Li, X.G. Li, B. Zhang, S.H. Song, X.J. Liu, X.L. Ye, Sci. Technol. Food Ind. (2013)

13. M.F. AlAjmi, M.T. Rehman, A. G.M. Rather, Int. J. Biol. Macromol. 116, 173-181 (2018)

14. K.S. Jani, D.S. Dalafave, Bioinform Biol. Insights 6, 23-31 (2012)

15. J. Shamsara, SpringerPlus. 5, 344 (2016)

16. J.S. Duca, V.S. Madison, J.H. Voigt, J. Chem. Inf. Model. 48, 659-668 (2008)

17. Z.P. Ke, Z.Z. Su, X.Z. Zhang, Z.Y. Cao, Y. Ding, L. Cao, G. Ding, Z.Z. Wang, H.C. Liu, W. Xiao, Bioorg. Med. Chem. Lett. 27, 3688-3692 (2017)

18. P. Towler, B. Staker, S.G. Prasad, S. Menon, J. Tang, T. Parsons, D. Ryan, M. Fisher, D. Williams, N.A. Dales, M.A. Patane, M.W. Pantoliano, J. Biol. Chem. 279, 17996-18007 (2004)

19. O. Trott, A.J. Olson, J. Comput. Chem. 31, 455-461 (2010)

20. Y.C. Jeong, M. Anwar, M.G. Moloney, Bioorg. Med. Chem. Lett. 24, 1190-1200 (2014)

21. P. Leeson, Nature 481, 455-461 (2012)

22. C.A. Lipinski, F. Lombardo, B.W. Dominy, P.J. Feeney, Adv. Drug Deliv. Rev. 23, 3-25 (1997)

23. D. Roosterman, C. Kempkes, G.S. Cottrell, B.E. Padilla, N.W. Bunnnett, C.W. Turck, M. Steinhoff, Endocrinology 149, 2200-2207 (2008)

24. J.H.; Ryu, J.H. Park, J.H. Eun, J.H. Jung, D.H. Sohn, Phytochemistry 44, 931-933 (1997) 
25. S.Y. Ko, K.J. Choi, K. Suzuki, Y. Suzuki, Chem. Pharm. Bull. 51, 404-408 (2003)

26. J.D. Park, D.K. Rhee, Y.H. Lee, Phytochem. Rev. 4, 159-175 (2005)

27. K.K. Li, C.M. Yao, X.W. Yang, Planta Med. 78, 189-192 (2012)

28. J.L. Yang, T.K. Ha, B. Dhodary, K.H. Kim, J. Park, C.L. Lee, Y.C. Kim, W.K. Oh, J. Nat. Prod. 77, 1615$1623(2014)$

29. K. Im, J. Kim, H. Min, J. Ginseng Res. 40, 309-314 (2016)

30. K. Kim, H.I. Jeong, I. Yang, S.J. Nam, KM. Lim, J. Ginseng Res. 2019

31. S.C. Kim, H.S. Kim, U.S. Patent (2019)

32. C.H. Lee, J.H. Kim, J. Ginseng Res. 38, 161-166 (2014)

33. S. Paik, J.H. Choe, G.E. Choi, J.E. Kim, J.M. Kim, G.Y. Song, E.K. Jo, Sci. Rep. 9, 4342 (2019)

34. X. Zheng, S. Wang, X. Zou, Y. Jing, R. Yang, S. Li, F. Wang, Exp. Anim. 66, 217-228 (2017)

35. G.R. Fenwick, J. Lutomski, C. Nieman, Food Chem. 38, 119-143 (1990)

36. A.S. Ammosov, V.I. Litvinenko, Pharm. Chem. J. 37, 83-94 (2003)

37. J.F. Hu, F.J. Shen, Nat. Prod. Res. Dev. 8, 77-91 (1996)

38. F. Rauchensteiner, Y. Matsumura, Y. Yamamoto, S. Yamaji, T. Tani, J. Pharm. Biomed. Anal. 38, 594$600(2005)$

39. Y. Zhou, M.K. Wang, X. Liao, X.M. Zhu, S.L. Peng, L.S. Ding, Chin. J. Anal. Chem. 32, 174-178 (2004)

40. G.M. Morris, R. Huey, W. Lindstrom, M.F. Sanner, R.K. Belew, D.S. Goodsell, A.J. Olson, J. Comput.

Chem. 19, 1639-1662 (2019)

41. Y.C. Jeong, M. Anwar, M.G. Moloney, Bioorg. Med. Chem. Lett. 24, 1901-1906 (2014)

\section{Tables}

Table 1. RMSD calculated between bioactive and docking conformations.

\begin{tabular}{lllllll}
\hline Inhibitor & ACE2 (1R4L) & \multicolumn{4}{l}{ ACE2 (2019-nCoV-RBD-ACE2) } \\
\hline & RMSD (nm) & Energy $(\mathrm{kcal} / \mathrm{mol})$ & $K_{\mathrm{i}}(\mu \mathrm{m} / \mathrm{L})$ & RMSD $(\mathrm{nm})$ & Energy $(\mathrm{kcal} / \mathrm{mol})$ & $K_{\mathrm{i}}(\mu \mathrm{m} / \mathrm{L})$ \\
MLN-4760 & 0.01 & -7.44 & 3.51 & 0.3 & -7.30 & 4.23 \\
\hline
\end{tabular}

Note: ${ }^{a}$ data of ACE2 from ref. [2]

Table 2. The identified compounds of the top 5\% selected compounds through docking scores and inhibition rete values of compounds for ACE2 kinase inhibition assay. 


\begin{tabular}{|c|c|c|c|c|}
\hline ID & Name & Source & $\begin{array}{l}\text { Gscore } \\
(\mathrm{kcal} / \mathrm{mol})\end{array}$ & $\begin{array}{l}\text { Inhibition } \\
(\%)\end{array}$ \\
\hline ZN00013 & Taraxasterol & $\begin{array}{l}\text { Cephalanoplos segetum } \\
\text { Bge.Kitam. }\end{array}$ & -9.2 & $\begin{array}{l}21.03 \pm \\
0.78\end{array}$ \\
\hline ZN00019 & Dioscin & Dioscorea nipponica Makino & -9.5 & - \\
\hline ZN00023 & Procyanidin C1 & Crataegus pinnatifida Bge. & -9.0 & - \\
\hline ZN00050 & 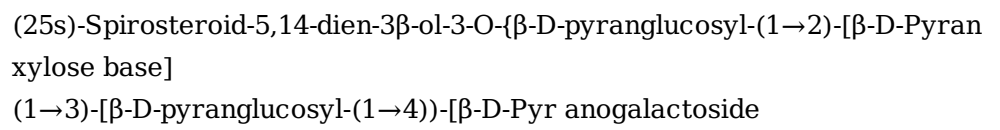 & $\begin{array}{l}\text { Polygonatum odoratum } \\
\text { Druce }\end{array}$ & -9.4 & - \\
\hline ZN00061 & Monoammonium glycyrrhizinate & Glycyrrhiza uralensis Fisch. & -9.8 & $\begin{array}{l}51.68 \pm \\
1.21\end{array}$ \\
\hline ZN00070 & Glycyrrhizic acid methyl ester & Glycyrrhiza uralensis Fisch. & -9.0 & $\begin{array}{l}50.40 \pm \\
1.78\end{array}$ \\
\hline ZN00104 & Lablab saponin I & Dolichos lablab L. & -9.6 & - \\
\hline ZN00105 & Soybean saponin $\mathrm{Bb}$ & Dolichos lablab L. & -9.4 & - \\
\hline ZN00131 & 3"'-O-Galloylmucic acid & Phyllanthus emblica Linn. & -9.0 & - \\
\hline ZN00172 & Jujuboside B & Ziziphus jujuba Mill. & -9.2 & - \\
\hline ZN00188 & Grosvenorine & Siraitia grosvenorii Swingle & -9.0 & - \\
\hline ZN00190 & Mogroside IV & Siraitia grosvenorii Swingle & -9.2 & - \\
\hline ZN00191 & Mogroside III & Siraitia grosvenorii Swingle & -9.8 & - \\
\hline ZN00192 & Mogroside IV & Siraitia grosvenorii Swingle & -9.0 & - \\
\hline ZN00202 & Siamenoside I & Siraitia grosvenorii Swingle & -9.2 & - \\
\hline ZN00309 & Desapioplatycodin D & $\begin{array}{l}\text { Platycodongrandif-1orum A. } \\
\text { DC }\end{array}$ & -9.3 & - \\
\hline ZN00325 & Arundoin & Lophatherum gracile Brongn. & -9.2 & $\begin{array}{l}43.75 \pm \\
0.41\end{array}$ \\
\hline ZN00339 & $\begin{array}{l}\text { (25R)-Snail steoids-5-alkene-3 } \beta, 17 \alpha \text {-glycol-3-O- } \beta \text {-D- pyranglucosyl }(1 \rightarrow 4) \text { - } \\
\beta \text {-D-pyranoside }\end{array}$ & Polygonati rhizoma & -9.4 & $\begin{array}{l}31.51 \pm \\
2.40\end{array}$ \\
\hline ZN00377 & Azukisaponin III & Sophora japonica L. & -9.0 & - \\
\hline ZN00378 & Azukisaponin I & Sophora japonica L. & -9.2 & $\begin{array}{l}18.71 \pm \\
1.62\end{array}$ \\
\hline ZN00397 & Jujuboside B1 & Semen Zizyphi Spinosae & -9.5 & - \\
\hline ZN00398 & Jujuboside D & Semen Zizyphi Spinosae & -9.0 & - \\
\hline ZN00438 & Cirenshenoside S & Ginseng & -9.1 & - \\
\hline ZN00439 & Pseudoginsenoside F11 & Ginseng & -9.1 & - \\
\hline ZN00441 & 20(S),24(R)-Ocotillol & Ginseng & -9.0 & $\begin{array}{l}81.62 \pm \\
1.08\end{array}$ \\
\hline ZN00445 & Ginsenoside Rg6 & Ginseng & -9.5 & $\begin{array}{l}60.70 \pm \\
2.90\end{array}$ \\
\hline ZN00451 & Ginsenoside F1 & Ginseng & -9.0 & - \\
\hline ZN00456 & Ginsenoside Rb3 & Ginseng & -9.6 & - \\
\hline ZN00471 & Ginsenoside Rg4 & Ginseng & -9.3 & - \\
\hline ZN00484 & vina-ginsenoside R4 & Ginseng & -9.3 & - \\
\hline ZN00489 & Pseudoginsenoside RT1 & Ginseng & -9.6 & - \\
\hline ZN00502 & Macranthoidin B & Lonicerae Flos & -9.3 & - \\
\hline ZN00503 & Macranthoside B & Lonicerae Flos & -9.0 & - \\
\hline
\end{tabular}

Table 3. The physiochemical properties of the top 5\% selected compounds through docking scores. 


\begin{tabular}{|c|c|c|c|c|c|c|c|c|}
\hline ID & MW & MSA & PSA & $\% \mathrm{PSA}^{\mathrm{a}}$ & $\mathrm{C} \log \mathrm{P}^{\mathrm{b}}$ & $C \log \mathrm{D}_{7.4}{ }^{\mathrm{c}}$ & $\mathrm{H}-\mathrm{D}^{\mathrm{d}} / \mathrm{H}-\mathrm{A}^{\mathrm{e}}$ & $\mathrm{RB}^{\mathrm{f}}$ \\
\hline ZN00013 & 426 & 788.1 & 20.2 & 2.6 & 7.45 & 7.45 & $1 / 2$ & 1 \\
\hline ZN00019 & 883 & 1334.6 & 235.6 & 17.7 & 2.01 & 2.01 & $8 / 16$ & 15 \\
\hline ZN00023 & 867 & 1040.5 & 331.1 & 31.8 & 4.44 & 4.41 & $15 / 18$ & 20 \\
\hline ZN00050 & 1015 & 1456.7 & 314.8 & 21.6 & -1.09 & -1.09 & $11 / 21$ & 15 \\
\hline ZN00061 & 821 & 1225.1 & 246.8 & 20.1 & 4.30 & -5.37 & $7 / 15$ & 14 \\
\hline ZN00070 & 837 & 1240.2 & 256.0 & 20.6 & 3.28 & -3.57 & $7 / 15$ & 15 \\
\hline ZN00104 & 1083 & 1568.2 & 347.6 & 22.2 & 0.65 & -2.87 & $11 / 22$ & 15 \\
\hline ZN00105 & 927 & 1412.3 & 274.8 & 19.5 & 2.18 & -1.25 & $10 / 17$ & 19 \\
\hline ZN00131 & 616 & 727.4 & 273.4 & 37.6 & 0.65 & 0.90 & $10 / 15$ & 16 \\
\hline ZN00172 & 1087 & 1642.4 & 314.8 & 19.2 & -0.39 & -0.39 & $11 / 21$ & 24 \\
\hline ZN00188 & 741 & 940.0 & 304.2 & 32.4 & 0.65 & -2.29 & $11 / 19$ & 19 \\
\hline ZN00190 & 1125 & 1677.9 & 397.5 & 23.7 & -2.70 & -2.70 & $16 / 24$ & 31 \\
\hline ZN00191 & 963 & 1468.7 & 318.4 & 21.7 & -0.93 & -0.93 & $13 / 19$ & 27 \\
\hline ZN00192 & 1450 & 2092.9 & 555.8 & 26.6 & -6.25 & -6.25 & $22 / 34$ & 31 \\
\hline ZN00202 & 1123 & 1690.7 & 388.3 & 22.9 & -2.75 & -2.75 & $16 / 23$ & 31 \\
\hline ZN00309 & 1093 & 1570.5 & 394.4 & 25.1 & -3.55 & -3.55 & 15.23 & 27 \\
\hline ZN00325 & 441 & 832.0 & 9.23 & 1.1 & 8.03 & 8.03 & $0 / 1$ & 2 \\
\hline ZN00339 & 739 & 1115.1 & 197.0 & 17.7 & 1.77 & 1.77 & $7 / 13$ & 12 \\
\hline ZN00377 & 811 & 1221.4 & 253.1 & 20.7 & 2.09 & -4.03 & $9 / 15$ & 17 \\
\hline ZN00378 & 781 & 1215.1 & 215.8 & 17.8 & 2.95 & -0.45 & $8 / 13$ & 14 \\
\hline ZN00397 & 1045 & 1546.0 & 314.8 & 20.4 & 0.68 & 0.68 & $11 / 21$ & 21 \\
\hline ZN00398 & 1207 & 1753.3 & 394.0 & 22.5 & -1.63 & -1.63 & $14 / 26$ & 27 \\
\hline ZN00438 & 1091 & 1585.8 & 374.1 & 23.6 & -1.47 & -1.47 & $14 / 22$ & 28 \\
\hline ZN00439 & 787 & 1237.2 & 228.2 & 18.4 & 1.02 & 1.02 & $9 / 14$ & 15 \\
\hline ZN00441 & 478 & 832.4 & 90.2 & 10.8 & 2.94 & 2.94 & $4 / 5$ & 6 \\
\hline ZN00445 & 753 & 1178.9 & 198.8 & 16.9 & 2.96 & 2.96 & $8 / 12$ & 16 \\
\hline ZN00451 & 622 & 1047.7 & 139.8 & 13.3 & 3.76 & 3.76 & $6 / 8$ & 13 \\
\hline ZN00456 & 1095 & 1638.9 & 377.3 & 23.0 & -2.23 & -2.23 & $15 / 23$ & 30 \\
\hline ZN00471 & 768 & 1191.4 & 218.9 & 18.4 & 1.86 & 1.86 & $9 / 13$ & 17 \\
\hline ZN00484 & 963 & 1470.0 & 318.4 & 21.7 & -1.09 & -1.09 & $13 / 19$ & 26 \\
\hline ZN00489 & 927 & 1379.1 & 291.8 & 21.2 & 1.74 & -1.65 & $10 / 17$ & 19 \\
\hline ZN00502 & 1400 & 2001.9 & 512.2 & 25.6 & -4.13 & -4.13 & $19 / 31$ & 31 \\
\hline ZN00503 & 1075 & 1586.5 & 353.9 & 22.3 & -0.09 & -2.69 & $13 / 22$ & 25 \\
\hline
\end{tabular}

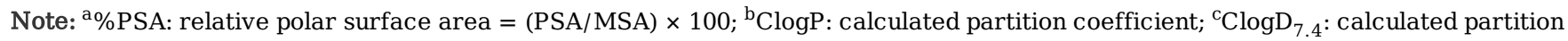
coefficient at pH 7.4; ${ }^{\mathrm{d}} \mathrm{H}-\mathrm{D}$ : hydrogen bond donor count; ${ }^{\mathrm{e}} \mathrm{H}-\mathrm{A}$ : hydrogen bond acceptor count; ${ }^{\mathrm{f}} \mathrm{RB}$ : rotatable bond count.

Abbreviations: MW: molecular weight; MSA: molecular surface area; PSA: polar surface area. 
Table 4. The molecular interaction between ACE2 and different inhibitors from the screened.

\begin{tabular}{|c|c|c|c|c|c|}
\hline ID/Structures & Molecular Interactions & Nature of Interactions & Distance $(\AA)$ & $\Delta G^{\mathrm{a}}(\mathrm{kcal} / \mathrm{mol})$ & $K_{\mathrm{d}}^{\mathrm{b}}\left(\mathrm{M}^{-1}\right)$ \\
\hline \multirow[t]{8}{*}{ ZN00061 } & ASN63:NH---Lig: O & Hydrogen bond & 2.90 & -1.63 & $6.23 \times 10^{2}$ \\
\hline & ASN63: O----Lig: $\mathrm{H}$ & Hydrogen bond & 2.17 & & \\
\hline & TYR510: H----Lig: O & Hydrogen bond & 3.06 & & \\
\hline & TYR50----Lig & Hydrophobic (Pi-Alkyl) & 3.15 & & \\
\hline & PHE504----Lig & Hydrophobic (Pi-Alkyl) & 3.50 & & \\
\hline & ARG273----Lig & Salt bridges & 4.74 & & \\
\hline & HIS345----Lig & Salt bridges & 4.70 & & \\
\hline & HIS505----Lig & Salt bridges & 3.35 & & \\
\hline \multirow{9}{*}{ ZN00070 } & SER-124: H----Lig: O & Hydrogen bond & 2.16 & -1.16 & 0.141 \\
\hline & TYR-199: H----Lig: O & Hydrogen bond & 1.99 & & \\
\hline & TRP-203: NH----Lig: O & Hydrogen bond & 1.68 & & \\
\hline & ASP-509: O---Lig: $\mathrm{H}$ & Hydrogen bond & 2.47 & & \\
\hline & TYR-510: H----Lig: O & Hydrogen bond & 2.97 & & \\
\hline & THR-125----Lig & Hydrophobic (Pi-Alkyl) & 3.28 & & \\
\hline & ASP-509----Lig & Hydrophobic (Pi-Alkyl) & 3.92 & & \\
\hline & LYS-187----Lig & Salt bridges & 4.09 & & \\
\hline & ARG-514---Lig & Salt bridges & 3.78 & & \\
\hline \multirow{11}{*}{ ZN00441 } & SER-124: H---Lig: O & Hydrogen bond & 2.12 & -4.72 & $3.45 \times 10^{4}$ \\
\hline & TYR-199: H----Lig: O & Hydrogen bond & 1.61 & & \\
\hline & TRP-203: H----Lig: O & Hydrogen bond & 2.50 & & \\
\hline & ASN-508: O----Lig: H & Hydrogen bond & 1.92 & & \\
\hline & ASP-509 H----Lig: O & Hydrogen bond & 2.66 & & \\
\hline & ASP-509: H----Lig: O & Hydrogen bond & 1.70 & & \\
\hline & TYR-510: O---Lig: H & Hydrogen bond & 1.87 & & \\
\hline & TYR-202----Lig & Hydrophobic (Pi-Alkyl) & 3.63 & & \\
\hline & ASN-508---Lig & Hydrophobic (Pi-Alkyl) & 3.76 & & \\
\hline & TYR-510----Lig & Hydrophobic (Pi-Alkyl) & 3.40 & & \\
\hline & TYR-510----Lig & Hydrophobic (Pi-Alkyl) & 3.54 & & \\
\hline \multirow[t]{10}{*}{ ZN00451 } & ASN-51 O---Lig: $\mathrm{H}$ & Hydrogen bond & 2.06 & -1.84 & $4.49 \times 10^{2}$ \\
\hline & ASN-51: H----Lig: O & Hydrogen bond & 1.75 & & \\
\hline & ASN-51 O---Lig: $\mathrm{H}$ & Hydrogen bond & 2.07 & & \\
\hline & TYR-127-----Lig & Hydrophobic (Pi-Alkyl) & 3.97 & & \\
\hline & TYR-127----Lig & Hydrophobic (Pi-Alkyl) & 3.41 & & \\
\hline & VAL-343----Lig & Hydrophobic (Pi-Alkyl) & 3.33 & & \\
\hline & HIS-345----Lig & Hydrophobic (Pi-Alkyl) & 3.19 & & \\
\hline & PHE-504----Lig & Hydrophobic (Pi-Alkyl) & 3,33 & & \\
\hline & PHE-504----Lig & Hydrophobic (Pi-Alkyl) & 3.29 & & \\
\hline & PHE-504----Lig & Hydrophobic (Pi-Alkyl) & 3.28 & & \\
\hline
\end{tabular}

Note: aDocking Binding Energy $(\Delta G)$; ' Docking Binding affinity $\left(K_{\mathrm{d}}\right)$.

\section{Figures}



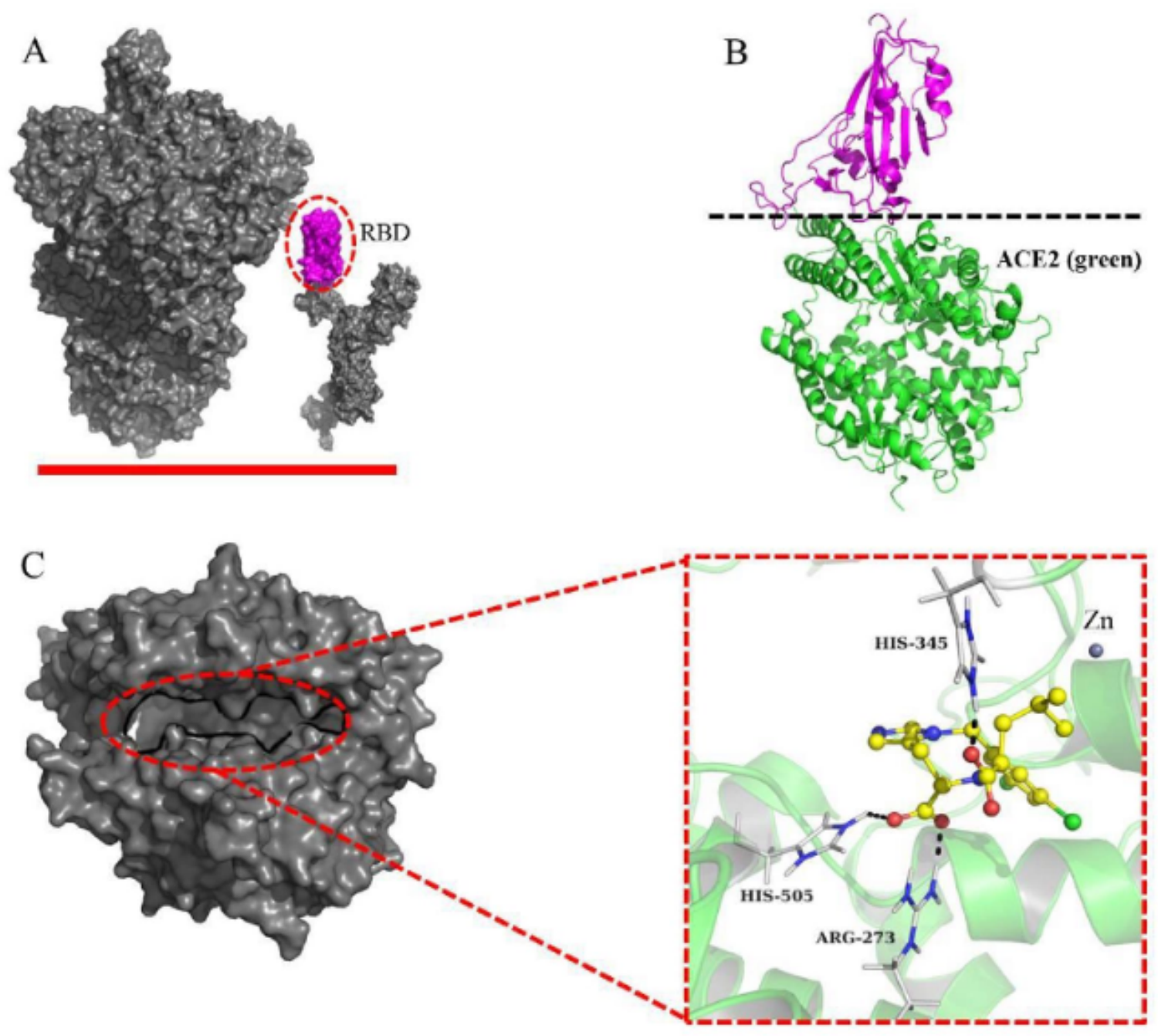

Figure 1

Stereo representations of interaction for ACE2 (2019-nCoV-RBD/ACE2) with MLN-4760. A. Structure of 2019-nCoV S and the receptor-binding domain (RBD) of S1 (subdomain 1), 2019-nCoV S and RBD were depicted by a surface model (grey). B. Overall structure of 2019-nCoV-RBD bong with (ACE2), the protein is shown as cartoon, RBD is colored magenta and ACE2 is colored green. C. Molecular docking model of MLN-4760 bound to the ACE2 (2019-nCoV-RBD/ACE2). The active site of ACE2 is shown as a surface model and the surface is colored grey. MLN-4760 is colored by element type ( $\mathrm{C}$, yellow; $\mathrm{O}$, red, polar $\mathrm{H}$, white), whereas key residue are shown as sticks ( $\mathrm{C}$, yellow; $\mathrm{O}$, red; $\mathrm{N}$, blue, polar $\mathrm{H}$, white) and hydrogen bonds are shown as black dashed lines. 


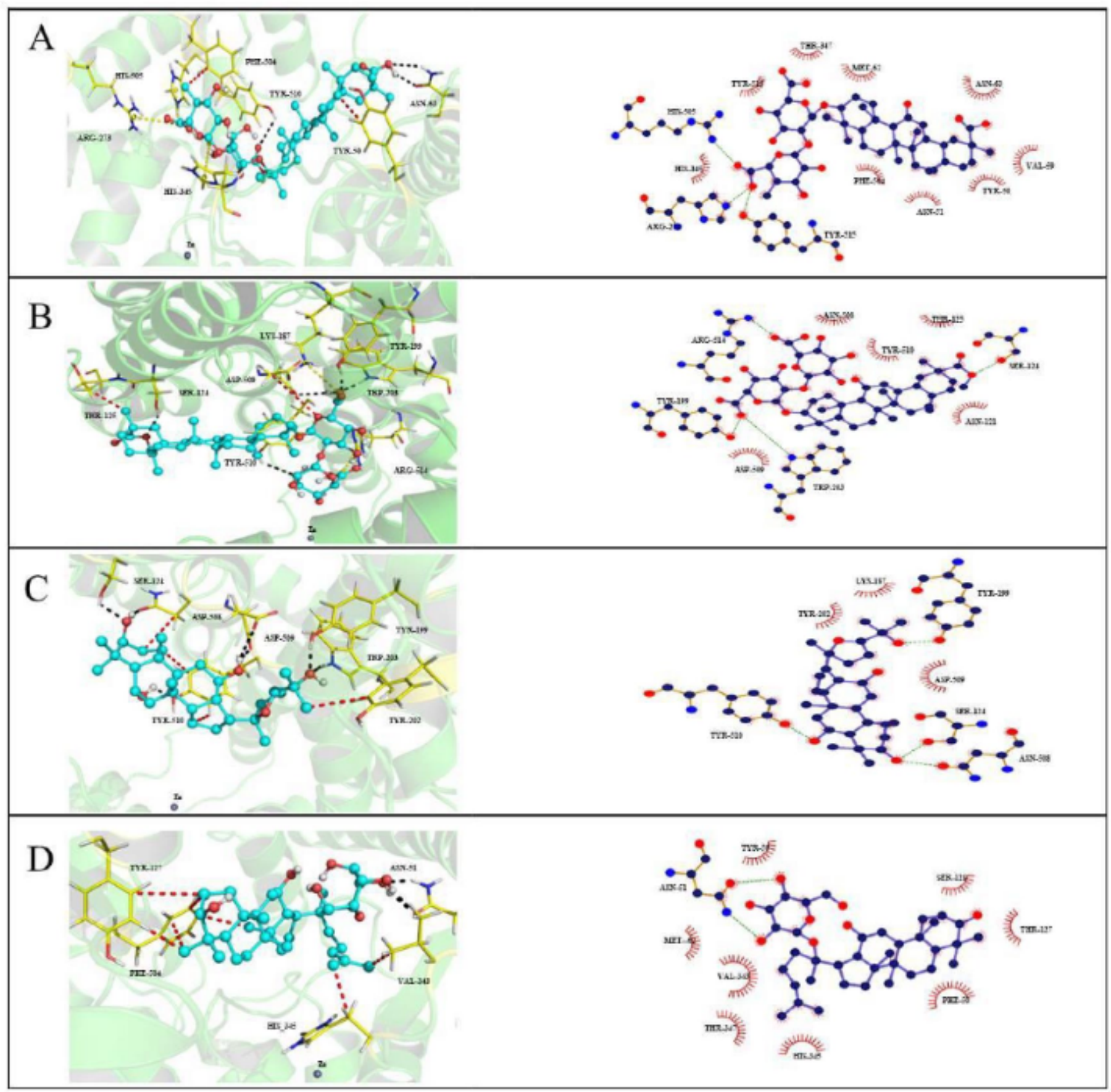

Figure 2

The stereo representation of interaction of ACE2 with monoammonium glycyrrhizinate (A), glycyrrhizic acid methyl ester (B), ginsenoside Rg6 (C) and ginsenoside F1 (D). The protein is shown as cartoon; ligand and the key residues are shown as sticks (ligand color: $\mathrm{C}$ cyan, $\mathrm{N}$ blue, $\mathrm{O}$ red and polar hydrogen); hydrogen bonds are shown as black dotted lines; hydrophobic interactions are shown as red dotted lines; salt bridges were shown as yellow dotted lines. The modes of interaction of compounds docking into the binding site of ACE2: Ligand and the key residues are shown as purple stick model; hydrogen bonds are shown as green dotted lines. 


\section{Supplementary Files}

This is a list of supplementary files associated with this preprint. Click to download.

- Electronicsupplementarymaterial.doc 